\title{
Erratum: Bekenstein inequalities and nonlinear electrodynamics [Phys. Rev. D 96, 125011 (2017)]
}

\author{
M. L. Peñafiel and F. T. Falciano
}

(Received 21 August 2018; published 30 August 2018)

DOI: 10.1103/PhysRevD.98.049902

In a recent paper we investigated the validity of Bekenstein's inequalities in the context of nonlinear electrodynamics (NLED). We concluded that Born-Infeld is a special case that fulfills all the requirements but generically NLED does not satisfy these inequalities. Our major result, namely, that in general NLED violates Bekenstein's inequalities, is robust but our analysis for the Born-Infeld case is wrong. Born-Infeld also violates Bekenstein's inequalities. In our paper, the inequality relating the total energy $\mathcal{E}_{\mathrm{BI}}$ and the total charge $Q$ of an isolated system is

$$
\mathcal{E}_{\mathrm{BI}}>\frac{Q^{2}}{8 \pi \mathcal{R}}=\frac{Q}{2} \phi_{M w}(\mathcal{R})
$$

where $\mathcal{R}$ and $\phi_{M w}(\mathcal{R})$ are respectively the radius of the smallest ball containing the system and the electric potential of a thin shell of radius $\mathcal{R}$ in Maxwell theory. Our proof is based on the mathematical fact that for a given value of electric field the Born-Infeld energy density is greater than or equal to the Maxwell energy density. However, for a given distribution of charged particles, Born-Infeld theory is less effective to produce electric fields than Maxwell's electrodynamics. Thus, Eq. (36) of our paper does not guarantee the above inequality. Indeed, one can show that a spherically symmetric solution violates this relation. The full inequality, Eq. (40), of our paper relating the energy, charge and angular momentum is also incorrect due to the violation of the conditions given by Eqs. (45) and (46). Notwithstanding, we would like to stress that theorem II is still correct. In Born-Infeld electrodynamics the quasilocal energy $\mathcal{E}_{\mathrm{BI}}(\Sigma)$ satisfies the inequality Eq. (37):

$$
\mathcal{E}_{\mathrm{BI}}(\Sigma) \mathcal{R} \geq\left|J_{\mathrm{BI}}(\Sigma)\right|
$$

where $J_{\mathrm{BI}}(\Sigma)$ is the angular momentum of the electromagnetic field with respect to the center of the smallest ball containing the system. General statements in NLED are hard to achieve due to the nonlinearity of the dynamical system. For the particular case of a spherically symmetric thin shell of radius $\mathcal{R}$, we can prove a modified inequality relating the total energy and charge of the system. Indeed, for a spherically symmetric solution, the electric displacement vector is $\mathbf{D}=\frac{\beta \lambda^{2}}{r^{3}} \mathbf{r}$ for $r>\mathcal{R}$ and $\lambda \equiv \sqrt{Q / 4 \pi \beta}$. Thus, the total energy $\mathcal{E}_{\mathrm{BI}}$ and electrostatic potential $\phi_{\mathrm{BI}}(\mathcal{R})$ read

$$
\begin{gathered}
\mathcal{E}_{\mathrm{BI}}=\int_{\mathbb{R}^{3}} \mathrm{~d}^{3} x \beta^{2}\left(\sqrt{1+D^{2} / \beta^{2}}-1\right)=4 \pi \beta^{2} \int_{\mathcal{R}}^{\infty} \mathrm{d} r\left(\sqrt{\lambda^{4}+r^{4}}-r^{2}\right), \\
\phi_{\mathrm{BI}}(\mathcal{R})=-\int_{\infty}^{\mathcal{R}} \mathrm{d} \mathbf{l} . \mathbf{E}=\beta \lambda^{2} \int_{\mathcal{R}}^{\infty} \frac{\mathrm{d} r}{\sqrt{\lambda^{4}+r^{4}}} .
\end{gathered}
$$

We can relate these two expressions by choosing $u=\sqrt{1+\lambda^{4} / r^{4}}-1, \mathrm{~d} v=r^{2} \mathrm{~d} r$ and integrating by parts to obtain

$$
\mathcal{E}_{\mathrm{BI}}=\frac{2 Q}{3}\left\{\frac{\mathcal{R}^{3} \beta}{2 \lambda^{2}}\left(1-\sqrt{1+\frac{\lambda^{4}}{\mathcal{R}^{4}}}\right)+\Phi_{\mathrm{BI}}(\mathcal{R})\right\}=\frac{Q}{2} \Phi_{\mathrm{BI}}(\mathcal{R})+\frac{Q \beta \lambda}{3} h\left(\frac{\mathcal{R}}{\lambda}\right),
$$

where we have defined $h(x)=\left(x^{3}-x \sqrt{x^{2}+1}\right)+\frac{1}{2 x} 2 F_{1}\left(\frac{1}{4}, \frac{1}{2} ; \frac{5}{4} ;-\frac{1}{x^{4}}\right)$ and ${ }_{2} F_{1}(\alpha, \beta ; \gamma ; x)$ is the hypergeometric function. In particular, $h(x)$ is always positive (see Fig. 1), hence the inequality

$$
\mathcal{E}_{\mathrm{BI}}>\frac{Q}{2} \Phi_{\mathrm{BI}}(\mathcal{R})
$$




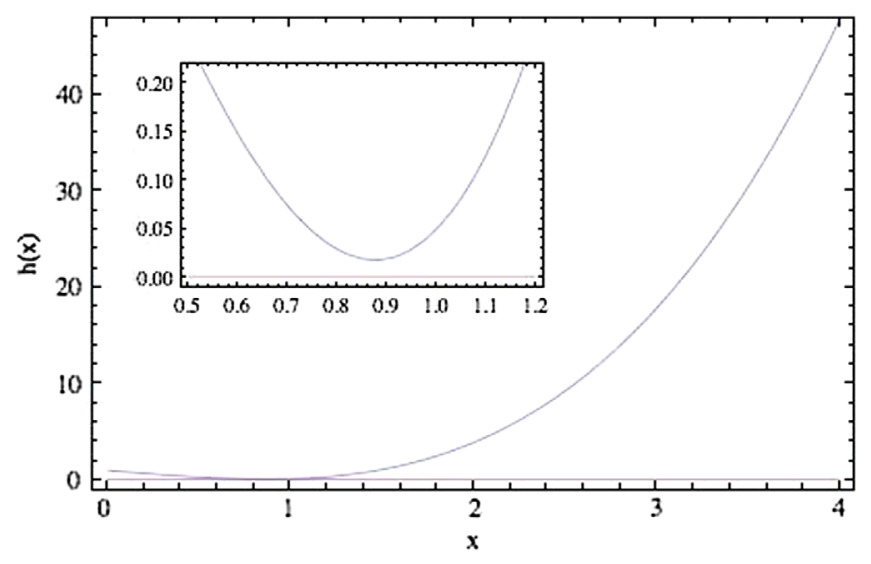

FIG. 1. Plot of the function $h(x)=\left(x^{3}-x \sqrt{x^{2}+1}\right)+\frac{1}{2 x} 2 F_{1}\left(\frac{1}{4}, \frac{1}{2} ; \frac{5}{4} ;-\frac{1}{x^{4}}\right)$.

holds for spherically symmetric solutions of Born-Infeld electrodynamics, which can be seen as a generalization of Eq. (1). Furthermore, since any Born-Infeld spherically symmetric configuration of constant charge density has an energy greater than the thin shell, the inequality also holds for any constant spherically symmetric charge distribution. 